\title{
SIMULATION BASED OPTIMIZATION OF JOINT MAINTENANCE AND INVENTORY FOR MULTI-COMPONENTS MANUFACTURING SYSTEMS
}

\author{
Abdullah Alrabghi \\ Ashutosh Tiwari \\ Manufacturing and Materials Department \\ Cranfield University \\ Cranfield, UNITED KINGDOM
}

\author{
Abdullah Alabdulkarim \\ Mechanical Engineering Department \\ Majmaah University \\ Majmaah, SAUDI ARABIA
}

\begin{abstract}
Maintenance and spare parts management are interrelated and the literature shows the significance of optimizing them jointly. Simulation is an efficient tool in modeling such a complex and stochastic problem. In this paper, we optimize preventive maintenance and spare provision policy under continuous review in a non-identical multi-component manufacturing system through a combined discrete event and continuous simulation model coupled with an optimization engine. The study shows that production dynamics and labor availability have a significant impact on maintenance performance. Optimization results of Simulated Annealing, Hill Climb and Random solutions are compared. The experiments show that Simulated annealing achieved the best results although the computation time was relatively high. Investigating multi-objective optimization might provide interesting results as well as more flexibility to the decision maker.
\end{abstract}

\section{INTRODUCTION}

Maintenance plays a vital role in sustaining and improving asset availability, which in turn affects the total output production of the system. A large and growing body of literature has investigated the optimization of maintenance in manufacturing systems. Dekker (1996) conducted a comprehensive review of maintenance optimization models and applications. It is interesting to note that simulation optimization was not mentioned in his research, rather, the focus was on mathematical modeling only.

As the complexity of maintenance systems has increased, the limitation of mathematical modeling became apparent as many maintenance policies are not analytically traceable (Garg and Deshmukh 2006, Nicolai and Dekker 2008). In a recent review, Sharma and Yadava (2011) discussed the published maintenance optimization models. They observed that the use of simulation has been an emerging trend which changed the maintenance view. This is mainly because simulation allows experimentation and better understanding of complex systems (Sebastian 2006).

Simulation based optimization (Rogers 2002) is an approach that consists of two main elements: a simulation model and an optimization engine. As shown in Figure 1, the optimization engine provides the simulation model with the decision variables. The simulation runs and provides the optimization engine with the objective function. Depending on the optimization algorithm the optimization engine will conduct an analysis and provide the simulation model with a new set of variables seeking to improve the objective function. This cycle will continue until a condition is met. For example a specific number of evaluations without improvement in the objective function. 


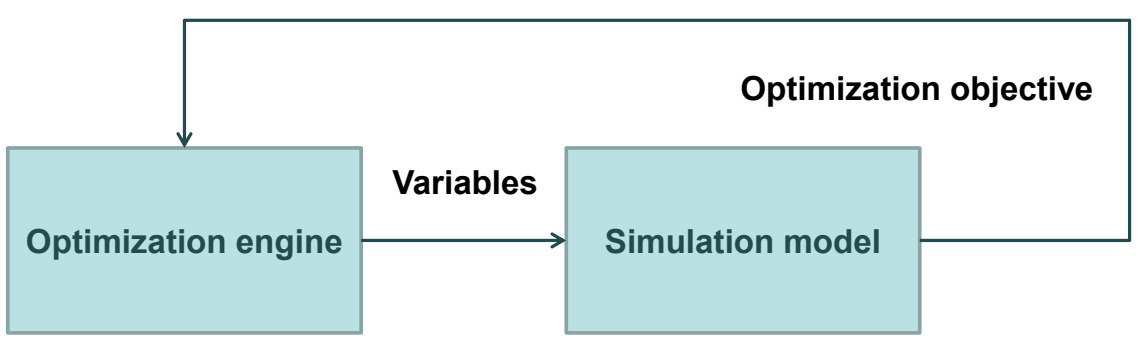

Figure 1: Simulation based optimization approach.

Alrabghi and Tiwari (2013) systematically classified the published literature on simulation based optimization in maintenance. Their study highlight that the vast majority of researchers optimize maintenance assuming maintenance resources are readily available including spare parts. However, research has consistently shown the significance of optimizing maintenance and spare parts jointly. Kabir and Farrash (1996) optimized maintenance jointly and separately with spare parts. Their study shows that separate optimization of maintenance and spare parts policies do not lead to global minimum costs. Similarly, the work of Sarker and Haque (2000) and Bruzzone and Bocca (2012) confirm this finding.

Van Horenbeek et al. (2012) review the literature on joint maintenance and optimization systems. Their study confirms the preference of simulation optimization to solve a problem with such a complex and stochastic nature. They observe that the research focus is on single-unit systems with few studies examining multi-components systems, usually assuming identical units. In addition, they suggest that simulation coupled with more sophisticated optimization techniques such as simulated annealing might decrease computational time and provide superior results. Figure 2 shows an overview classification of published literature and the focus of this research is illustrated by the shaded boxes.

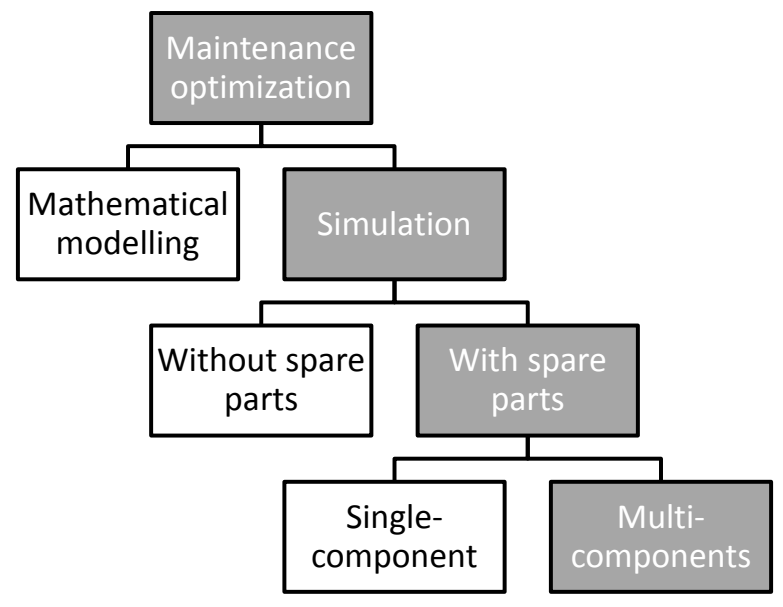

Figure 2: Classification of published literature and the focus of this research.

Attempts to optimize maintenance and spare parts in multi-component systems through simulation optimization are shown in Table 1. Sarker and Haque (2000) examined a manufacturing system comprising of identical units and compared optimization results jointly with spare parts policies and separately. In fact, with the exception of Ilgin and Tunali (2007), all remaining studies assumed units to be identical and independent which does not reflect the complexity of real manufacturing systems. Similarly, it is apparent that studies assumed that maintenance technicians are always available. Discrete event simulation was expected to dominate as it is the most popular technique in modeling manufacturing systems (Jahangirian 
et al. 2010). Studies either used a single optimization algorithm (Chen, Hsu, and Chen 2006; Ilgin and Tunali 2007) or conducted design of experiments (Sarker and Haque 2000; Boulet, Gharbi, and Kenn 2009). Table 1 also shows how the present study complements the previous research by the inclusion of labor, modeling through combined discrete event and continuous simulation and examining three optimization algorithms.

Table 1: Review of simulation based optimization of joint maintenance and spare parts in multicomponents systems.

\begin{tabular}{|l|l|l|l|l|}
\hline Paper & Non-identical & Labor & Simulation technique & Optimization algorithm \\
\hline $\begin{array}{l}\text { (Sarker and } \\
\text { Haque 2000) }\end{array}$ & No & No & Discrete event & Design of experiment \\
\hline $\begin{array}{l}\text { (Chen, Hsu, and } \\
\text { Chen 2006) }\end{array}$ & No & No & Discrete event & Scatter search \\
\hline $\begin{array}{l}\text { (Ilgin and Tunali } \\
\text { 2007) }\end{array}$ & Yes & No & Discrete event & Genetic algorithms \\
\hline $\begin{array}{l}\text { (Boulet, Gharbi, } \\
\text { and Kenn 2009) }\end{array}$ & No & No & Discrete event & Design of experiment \\
\hline $\begin{array}{l}\text { Current research } \\
\text { Yes }\end{array}$ & Yes & $\begin{array}{l}\text { Combined discrete event } \\
\text { and continuous }\end{array}$ & $\begin{array}{l}\text { Simulated Annealing, Ran- } \\
\text { dom Solutions and Hill } \\
\text { Climb }\end{array}$ \\
\hline
\end{tabular}

The aim of this paper is to explore specific gaps observed in the previous literature by optimizing preventive maintenance and spare provision policy under continuous review in a non-identical multicomponent manufacturing system through a combined discrete event and continuous simulation model coupled with an optimization engine. Three optimization algorithms are compared and examined, namely, Simulated Annealing, Random Solutions and Hill Climb.

\section{PROBLEM DEFINITION}

Notations:

$\begin{array}{ll}M c_{n} & \text { Machine } \mathrm{n} \\ S P_{n} & \text { Spare part for machine } \mathrm{n} \\ S_{n} & \text { Reorder level for } \mathrm{SP}_{\mathrm{n}} \\ Q_{n} & \text { Order quantity for } \mathrm{SP}_{\mathrm{n}} \\ P M \text { freq }_{n} & \text { Preventive maintenance frequency for machine } \mathrm{n} \\ \text { Labor } & \text { Number of maintenance technicians }\end{array}$

Preventive Maintenance (PM) costs less than Corrective Maintenance (CM) and requires considerably shorter periods. Unlike CM, PM is scheduled therefore it can be accommodated in both production and maintenance plans. The main question is how often a machine should be maintained (see Figure 3). Overmaintaining machines leads to unnecessary halts and costs. On the other hand, under-maintaining leads to unplanned breakdowns that results in chaos and expensive impacts. 
Alrabghi, Tiwari, and Alabdulkarim
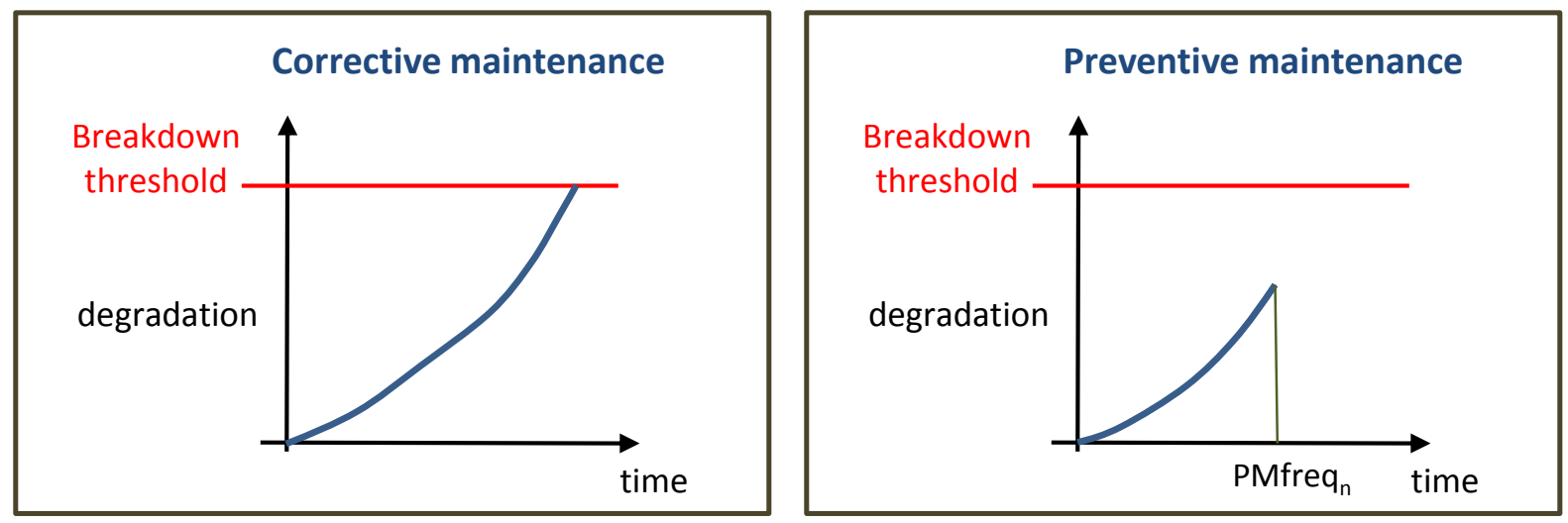

Figure 3: Comparison of CM and PM.

In theory, the most effective solution is to schedule PM to be conducted just before a machine breaks down. In practice that is impossible partly because the machine degradation is a stochastic process. Other challenges include production dynamics in multi-component systems. While PM is planned through a fixed time frame, the machine degradation increases only when it is in use. However, the machine could become idle if there are not any parts to be processed as a result of another breakdown that occurred in the production line.

The problem becomes more complex when considering spare parts and labor availability. Spare parts availability affect maintenance performance considerably. Shortage of spare parts increases machines unavailability. On the other hand, keeping a large inventory of spare parts might lead to unnecessary costs. One of the methods to control inventory is the continuous review spare provision policy includes $(s, Q)$ where $Q$ units are ordered each time the stock level reaches $s$.

In this study, we investigate a non-identical multi-component manufacturing system with the aim of optimizing joint preventive maintenance and spare provision policy under continuous review $(s, Q)$ through simulation based optimization, namely a combined discrete event and continuous simulation model coupled with an optimization engine.

\section{SIMULATION MODEL}

\subsection{Simulation Technique}

A combined discrete event and continuous simulation model is developed using the available simulation package within the research group: Witness 12 manufacturing performance edition, which is a product of Lanner Group. Manufacturing systems are in general discrete event systems where the state of the system changes according to events (Sebastian 2006). Additionally, discrete event simulation has been successfully applied to model various types of manufacturing systems including maintenance systems (Jahangirian et al. 2010). Continuous simulation, on the other hand, is used to model systems that change continuously over time (Robinson 2007). This is particularly true for the machine degradation process where machines deteriorate continuously over time when in use.

\subsection{Overview of the Manufacturing System}

A model of a manufacturing system consisting of 6 non-identical machines is developed as illustrated in Figure 4. A buffer exists after the following machines: machine 1, machine 2, machine 3 and machine 4, and machine 5. Parts are pulled into the manufacturing system through machine 1 and machine 2 in parallel. The output of machine 1 is stored in a buffer until machine 3 becomes available. Similarly, the output of machine 2 is stored in a buffer until machine 4 becomes available. The output of both machine 3 
and machine 4 is stored in a buffer and is assembled in machine 5 when it becomes available. Finally, the output of machine 5 is stored in a buffer until machine 6 becomes available which then ships the final product out of the system.

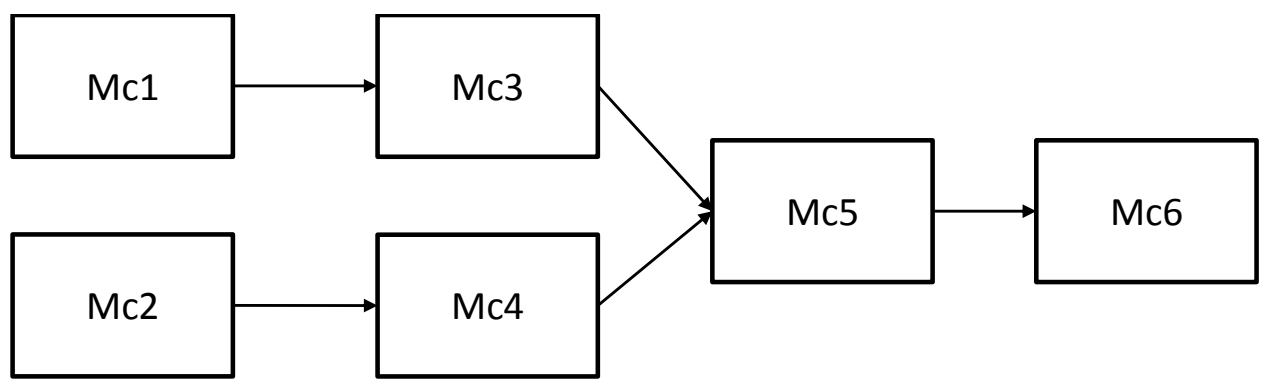

Figure 4: Overview of the simulation model.

Failure patterns for machines are assumed to follow Weibull and Exponential, two of the most widely used distributions to model lifetime in reliability and maintenance engineering (Ben-Daya 2009). Cycle times follow Triangular distribution and vary between machines. Repair times for CM and PM tasks follow a Uniform distribution and vary between machines as well. All related distributions along with their parameters are shown in Table 2.

Table 2: Cycle times, breakdown patterns and repair times for the manufacturing system.

\begin{tabular}{|l|l|l|l|l|}
\hline Machine & Cycle time & Breakdown pattern & CM duration & PM duration \\
\hline Mc1 & Triangle $(3,6,12)$ & Weibull $(2,3)$ & IUniform $(1,3)$ & IUniform $(0.2,1)$ \\
\hline Mc2 & Triangle $(4,5,11)$ & Weibull $(4,2)$ & IUniform $(1.2,3.5)$ & IUniform $(0.8,2.5)$ \\
\hline Mc3 & Triangle $(3,9,10)$ & Weibull $(2,2.5)$ & IUniform $(1.7,2.3)$ & IUniform $(1,1.5)$ \\
\hline Mc4 & Triangle $(5,9,10)$ & Weibull $(3,1)$ & IUniform $(1.5,3)$ & IUniform $(1,1.5)$ \\
\hline Mc5 & Triangle $(7,9,13)$ & NegExp $(2.5)$ & IUniform $(0.7,2.5)$ & IUniform $(0.5,1.6)$ \\
\hline Mc6 & Triangle $(5,10,14)$ & NegExp (3) & IUniform $(1,2.2)$ & IUniform $(0.4,1.8)$ \\
\hline
\end{tabular}

Spare provision policy is under continuous review and it includes $(s, Q)$ where $Q$ units are ordered each time the stock level reaches $s$. Lead times are stochastic and follow a uniform(72, 168) distribution.

All machines are subject to $\mathrm{CM}$ when their degradation reaches a specific threshold. They are also subject to PM at predetermined intervals $\left(P M f r e q_{n}\right)$. PM and CM cannot occur at the same time. If a machine is broken down, all PM activities will be postponed until the machine is fixed. On the other hand, if a machine is undergoing preventive maintenance it will not be working and thus its degradation level remains constant. Maintenance tasks are assumed to be perfect where the machine becomes as good as new after maintenance actions. In addition, machines are assumed to deteriorate only when in use.

A typical machine degradation cycle is shown in Figure 5. The machine will degrade as long as it is in use. If there are not any parts to be processed due to the breakdown of a preceding machine or due to shortages of raw materials, the machine will become idle and hence its degradation level remains constant. If the degradation level reaches the breakdown threshold the machine will stop working instantly and it will be repaired as soon as there are available spare parts and labor. Repair time for CM is always longer than the repair time for PM as shown above in Table 2. On the other hand, PM is conducted periodically every Pmfreq ${ }_{n}$ unit of time. Observing machine degradation in the simulation leads to the conclusion that production dynamics and labor availability have a significant impact on maintenance performance. 


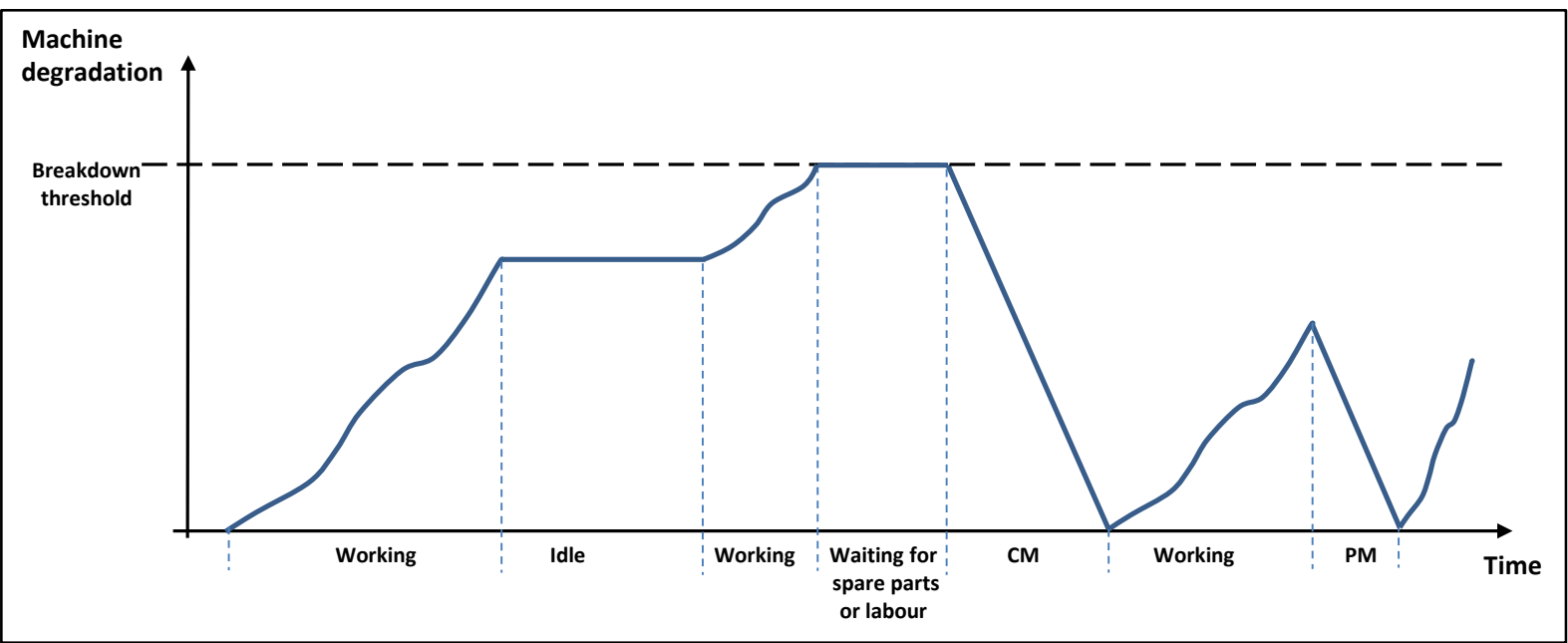

Figure 5: A typical machine degradation cycle.

\subsection{Cost Formulation}

Total Cost can be divided into three subcategories as follows:

where

Total Cost= maintenance cost + spare parts cost + unavailability cost

Maintenance cost $=P M$ cost $+C M$ cost, and, Spare parts cost $=$ order cost + holding cost .

Maintenance cost includes the cost of both preventive and corrective maintenance tasks and is incurred whenever these tasks are executed. The cost of spare parts includes the cost of ordering which is incurred whenever the inventory for $\mathrm{SP}_{\mathrm{n}}$ falls below the reorder level $\mathrm{s}_{\mathrm{n}}$ and the cost of holding which is incurred for every unit of time a spare part spends in the inventory. On the other hand, unavailability cost is incurred for every time unit a machine is not available due to maintenance tasks, shortage of spare parts or waiting for labor. The costs are constant during the simulation and are as follows:

- $\quad$ Corrective maintenance $=2000 /$ task,

- Preventive maintenance $=750 /$ task,

- Holding cost $=2 /$ unit/hour,

- $\quad$ order cost $=100 /$ order,

- Unavailability penalty $=300 /$ unavailable machine hour.

Hours were considered to be the time units for the simulation. The model was run for 10 years $=$ 87600 hours with a warm up period of 1 year $=8760$ hours and 3 replications.

\section{OPTIMIZATION PROBLEM}

\subsection{Problem Formulation}

A single objective optimization of both preventive maintenance and spare parts is formulated. The objective is minimizing the Total Cost as detailed in the previous section. 19 decision variables are defined for this problem as follows:

$$
\begin{gathered}
1<\text { labor }<6, \\
168 \text { (1 week) }<\text { Pmfreqn }<504 \text { (3 weeks), } \\
0<\text { sn }<15, \\
1<Q n<15 .
\end{gathered}
$$




\subsection{Optimization Algorithms}

Hill Climb is a local search heuristic algorithm that changes a single element in each iteration depending on the objective function performance. The main advantage of this algorithm is that it uses little memory and it makes rapid progress. However, one of its known limitations is that it performs local rather than global search. This will likely result in local optimum solution while there might be a better global solution as show in Figure 6 (Russell and Norvig 2003).

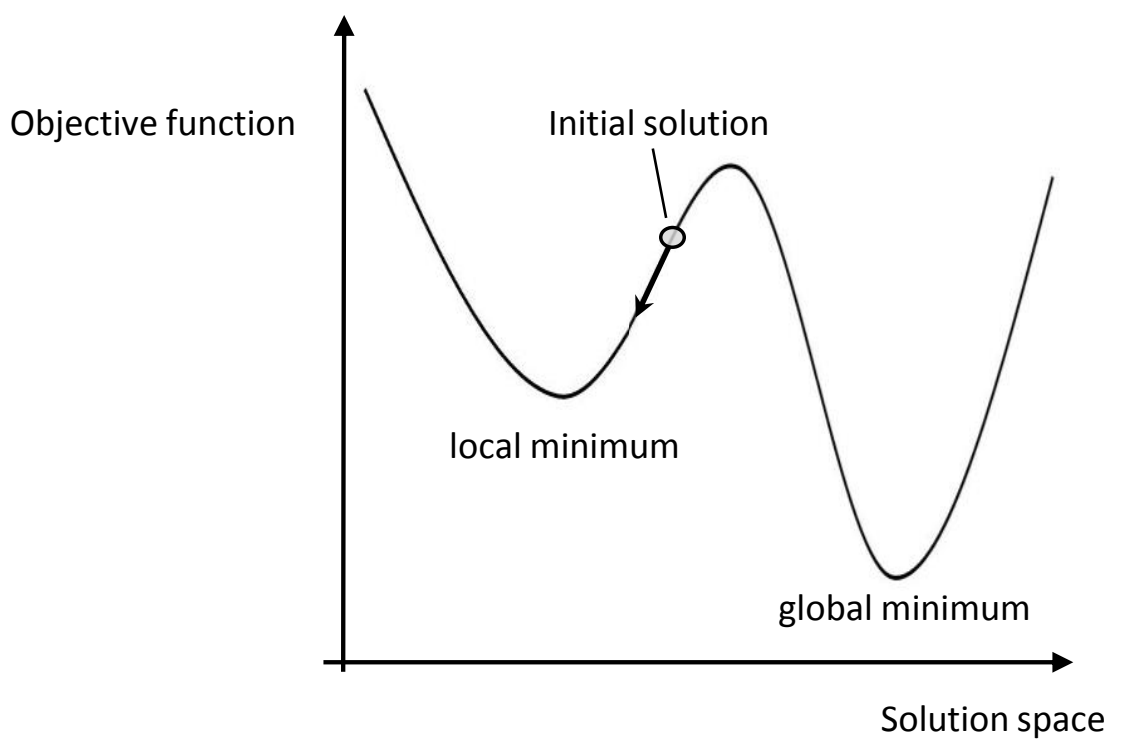

Figure 6: Global and local optimum solutions. Adapted from Russell and Norvig (2003).

In contrast, Random Solutions is simply randomizing the values of decision variables without a structured algorithm to guide it to better solutions. This search method can search globally but without the capability to learn from evaluations. Therefore it is seen as inefficient and unlikely to result in global optimum. It is used to find initial feasible solutions for complex problems (Deb 2005).

Simulated annealing (SA) combines the advantages of both Hill Climb and Random Solutions. The concept comes from the annealing process in metallurgy to harden metals. Metals are melted in high temperature at the start and then cooled gradually in a controlled environment. In simulated annealing, the algorithm starts with high randomized search and builds on good solutions. However the randomization gradually decreases as the algorithm learns and the focus is switched to finding a local improvement to the best solution so far (Russell and Norvig 2003).

Preliminary analysis was conducted by running the optimization several times while changing the number of evaluation for each algorithm continuously and monitoring the performance. It is observed that all three algorithms struggle to improve the objective function after around 150 evaluations. Therefore the number of evaluations was set to 200 for all algorithms. Experiments were run using Witness optimizer version 5 which is a product of Lanner Group.

\section{RESULTS AND DISCUSSIONS}

Optimization results are presented in Table 3. Experiments were run on PC with Intel Core i7-2600 CPU (a) $3.40 \mathrm{GHz}$. Simulated annealing achieved the best result providing 13\% and 2\% reduction in cost compared to Hill Climb and Random Solution respectively. Depending on the maintenance expenditures, the savings can be significant. It is interesting to note that Random solution yielded better results than Hill 
Climb. This can be due to the vast search area containing many optimum solutions where Hill Climb is not capable of searching beyond neighboring solutions as previously illustrated in Figure 6.

Table 3: Optimization computation time and best results.

\begin{tabular}{|c|l|l|l|}
\hline \multicolumn{2}{|l|}{ Optimization algorithm } & $\begin{array}{l}\text { Computation time } \\
\text { (hh:mm:ss) }\end{array}$ & Best result \\
\hline 1 & Random solutions & $07: 18: 52$ & $26,563,700$ \\
\hline 2 & Hill Climb & $04: 05: 01$ & $29,893,939$ \\
\hline 3 & $\begin{array}{l}\text { Adaptive Thermostatistical SA (Simu- } \\
\text { lated Annealing) }\end{array}$ & $08: 17: 34$ & $25,958,218$ \\
\hline
\end{tabular}

On the other hand, simulated annealing performed poorly in terms of computational time requiring almost 100\% and 12.5\% more time compared to Hill Climb and Random Solution respectively. In general, all optimization algorithms required a significant time to produce the results which is an indicative of the problem complexity. Optimizing a larger manufacturing system means adding more machines and thus more decision variables which would inevitably increase the required computational time exponentially. Conducting design of experiments before running the optimization might assist in focusing the optimization effort in the factors that have the most impact on the objective function and hence reduce the computational time.

Figure 7 shows the performance of the three optimization algorithms. It seems that both Simulated annealing and Hill Climb have a similar convergence rate at the beginning while Random Solutions converged at a faster rate. Furthermore, Hill Climb and Random Solutions struggled to deliver significant improvements early in the process, after around the $15^{\text {th }}$ evaluation. On the other hand, Simulated annealing continued to converge and provide apparent improvements until around the $135^{\text {th }}$ evaluation.

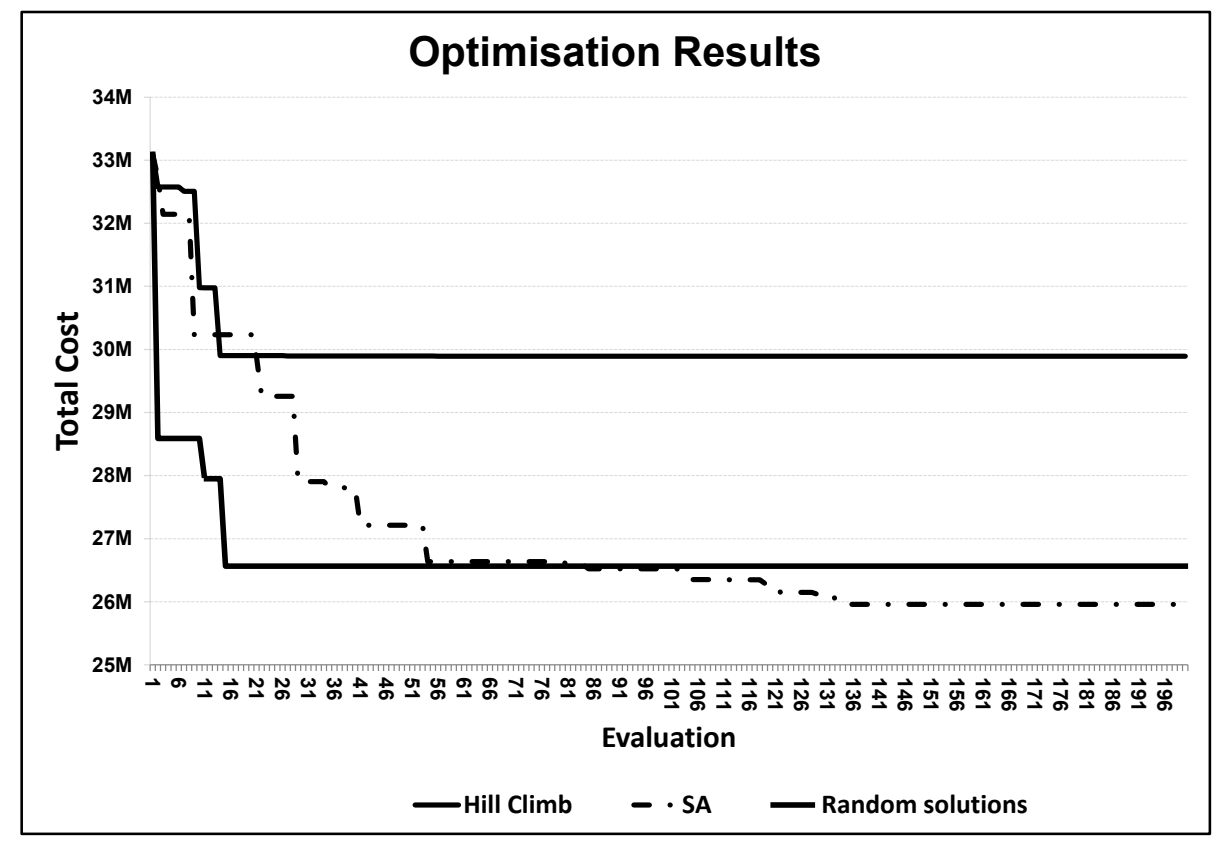

Figure 7: Algorithms' performance over evaluations. 
The optimal decision variables are compared in Table 4. It is interesting to observe that although the Total Cost for both SA and Random solutions is almost similar, the optimal variables differed substantially. This might suggest that there are multiple optimum solutions. Additionally, this might indicate that there is still room for improvements, perhaps by altering SA parameters or by experimenting with other optimization algorithms such as Genetic Algorithms (Deb 2005).

Table 4: Optimal decision variables.

\begin{tabular}{|l|r|r|r|}
\hline & Hill Climb & $\begin{array}{l}\text { Random So- } \\
\text { lutions }\end{array}$ \\
\hline Total Cost & $29,893,939$ & $26,563,705$ & $25,958,218$ \\
\hline PMFreq $_{1}$ & 209 & 262 & 308 \\
\hline PMFreq $_{2}$ & 203 & 502 & 213 \\
\hline PMFreq $_{3}$ & 358 & 216 & 459 \\
\hline PMFreq $_{4}$ & 393 & 324 & 374 \\
\hline PMFreq $_{5}$ & 384 & 422 & 373 \\
\hline PMFreq $_{6}$ & 341 & 281 & 322 \\
\hline Labor $_{s_{1}}$ & 4 & 3 & 3 \\
\hline$s_{2}$ & 0 & 5 & 9 \\
\hline$s_{3}$ & 8 & 2 & 7 \\
\hline$s_{4}$ & 2 & 12 & 2 \\
\hline$s_{5}$ & 2 & 11 & 1 \\
\hline$s_{6}$ & 10 & 4 & 13 \\
\hline$Q_{1}$ & 9 & 7 & 9 \\
\hline$Q_{2}$ & 11 & 6 & 10 \\
\hline$Q_{3}$ & 3 & 9 & 4 \\
\hline$Q_{4}$ & 7 & 11 & 8 \\
\hline$Q_{5}$ & 12 & 7 & 2 \\
\hline$Q_{6}$ & 13 & 10 & 5 \\
\hline & 6 & 10 & 6 \\
\hline & & & \\
\hline
\end{tabular}

However, with a single model, caution must be applied, as the findings might not be transferable to other maintenance systems. More experiments are needed to understand optimization algorithms performances, preferably with different maintenance models such as condition based maintenance and imperfect maintenance.

\section{CONCLUSIONS}

Preventive maintenance and spare provision policy were optimized using a combined discrete event and continuous simulation model coupled with an optimization engine. The results illustrate the complexity of optimizing maintenance in non-identical multi-component manufacturing systems. The study also shows that production dynamics and labor availability have a significant impact on maintenance performance. Simulated annealing produced better results compared with Random Solutions and Hill Climb although the computation time was relatively high.

Further work needs to be done to establish whether computational expenses can be reduced whilst improving the objective function, possibly through design of experiments, experimenting with different 
SA parameters or investigating Genetic Algorithms. Further research might also explore multi-objective optimization of both cost and availability as it has the ability to diverge and produce a range of results providing more flexibility to the decision maker.

\section{ACKNOWLEDGMENT}

The authors would like to thank the Ministry of Higher Education in Saudi Arabia for funding this research.

\section{REFERENCES}

Alrabghi, A., and A. Tiwari. 2013. "A Review of Simulation-Based Optimisation in Maintenance Operations." In Proceedings of the 2013 UKSim 15th International Conference on Computer Modelling and Simulation, 353-358, Cambridge, IEEE, 10-12 April 2013.

Ben-Daya, M. 2009. "Failure Statistics." In Handbook of Maintenance Management and Engineering, edited by M. Ben-Daya, J. Knezevic, Abdul Raouf, D. Ait-Kadi, and S. O. Duffuaa. London: Springer London.

Boulet, J. F., A. Gharbi, and J. P. Kenn. 2009. "Multiobjective Optimization in an Unreliable FailureProne Manufacturing System." Journal of Quality in Maintenance Engineering 15:397-411.

Bruzzone, A., and E. Bocca. 2012. "Innovative Solutions Based on Simulation Combined with Optimization Techniques for Maintenance Service Management within Complex Systems." International Journal of Modeling, Simulation, and Scientific Computing 3:1-23.

Chen, M., C. Hsu, and S. Chen. 2006. "Optimizing Joint Maintenance and Stock Provisioning Policy for a Multi-Echelon Spare Part Logistics Network." Journal of the Chinese Institute of Industrial Engineers 23:289-302.

Deb, K. 2005. Optimization for Engineering Design : Algorithms and Examples. New Delhi: PrenticeHall of India Private Limited.

Dekker, R. 1996. "Applications of Maintenance Optimization Models: A Review and Analysis." Reliability Engineering and System Safety. 52:229-240.

Garg, A., and S.G. Deshmukh. 2006. "Maintenance Management: Literature Review and Directions." Journal of Quality in Maintenance Engineering 12:205-238.

Ilgin, M., and S. Tunali. 2007. "Joint Optimization of Spare Parts Inventory and Maintenance Policies using Genetic Algorithms." The International Journal of Advanced Manufacturing Technology 34:594604.

Jahangirian, M., T. Eldabi, A. Naseer, L. Stergioulas, and T. Young. 2010. "Simulation in Manufacturing and Business: A Review." European Journal of Operational Research 203:1-13.

Nicolai, R. P., and R. Dekker. 2008. "Optimal Maintenance of Multi-Component Systems: A Review." In Complex System Maintenance Handbook, edited by K. Kobbacy, and D. N. Murthy, 263-286. London: Springer.

Robinson, S. 2007. Simulation: The Practice of Model Development and Use. Chichester: Wiley.

Rogers, P. 2002. "Optimum-Seeking Simulation in the Design and Control of Manufacturing Systems: Experience with OptQuest for Arena." In Proceedings of the 1994 Winter Simulation Conference, Edited by J. D. Tew, S. Manivannan, D. A. Sadowski, and A. F. Seila, 1142 - 1150. Piscataway, New Jersey: Institute of Electrical and Electronics Engineers, Inc.

Russell, S., and P. Norvig. 2003. Artificial Intelligence : A Modern Approach. Upper Saddle River, N.J.: Prentice Hall/Pearson Education.

Sarker, R., and A. Haque. 2000. "Optimization of Maintenance and Spare Provisioning Policy using Simulation." Applied Mathematical Modelling 24: 751-760.

Sebastian, W. 2006. "Simulation of Manufacturing Processes." In Electronics Process Technology Production Modelling, Simulation and Optimisation, edited by W. Sauer, 119-172. London: Springer. 
Sharma, A., G.S. Yadava, and S.G. Deshmukh. 2011. "A Literature Review and Future Perspectives on Maintenance Optimization." Journal of Quality in Maintenance Engineering 17:5-25.

Horenbeek, A., J. Buré, D. Cattrysse, L. Pintelon, and P. Vansteenwegen. 2012. "Joint Maintenance and Inventory Optimization Systems: A Review." International Journal of Production Economics 143:499-508.

Zohrul Kabir, A.B.M., and S.H.A. Farrash. 1996. "Simulation of an Integrated Age Replacement and Spare Provisioning Policy using SLAM." Reliability Engineering \& System Safety 52:129-138.

\section{AUTHOR BIOGRAPHIES}

ABDULLAH ALRABGHI is a PhD researcher in Manufacturing and Materials Department at Cranfield University, UK. His research interest are in industrial/manufacturing engineering. His current work examines simulation based optimization of maintenance operations in manufacturing systems. He obtained his MSc in Engineering Business Management from University of Warwick, UK and his BSc in Industrial Engineering from King Abdulaziz University, Saudi Arabia. His email address is a.alrabghi@cranfield.ac.uk.

ASHUTOSH TIWARI is currently a Professor of Manufacturing Informatics and Head of the Product and Service Innovation Centre at Cranfield University .He gained his $\mathrm{PhD}$ in multi-criteria optimization from Cranfield University, UK. His research focus is on the application of computing techniques to process re-design/optimization. He has published extensively in his research career with around 150 research publications. He was awarded the Institution of Mechanical Engineers) IMechE (Thatcher Bros Prize 2008/09 for the best journal paper in manufacturing.His email address is a.tiwari@cranfield.ac.uk.

ABDULLAH A. ALABDULKARIM is a lecturer in industrial systems simulation at Majmaah University in Saudi Arabia. Currently is a PhD candidate at Cranfield University, UK. His research focuses on simulation modelling for service sectors. He obtained his MSc in Logistics and Optimization from University of Portsmouth, and his BSc in Industrial Engineering was obtained from King Saud University. His background is from the aerospace industry in aviation maintenance and operations. He worked for several industries before pursuing his academic career. His email address is a.alabdulkarim@mu.edu.sa. 